\title{
Drawing Robot using Firebird V
}

\author{
M. V. Sreenivas Rao ${ }^{1}$, M Shiva Kumar ${ }^{2}$ \\ Assoc Prof, Dept. of EIE, GSSSIETW, Mysuru ${ }^{1}$ \\ Professor \& Head, Dept of EIE, GSSSIETW, Mysuru²
}

\begin{abstract}
The objective of this project is to design the drawing bot using "Fire Bird V ATMEGA2560" microcontroller. Drawing robot is the robot that will draw with a regular marking pen on a piece of paper. The project focuses on drawing a geometric shape. Drawing robot is a application which creates the drawing using firebird V through MatLab. Execution in MatLab yields the result in binary matrix .To move the robot, the program is executed in the AVR studio and result is obtained as Hex file. The Hex file is burn to the robot through USB cable for the movement of robot to draw an image .This project helps us to perform task faster with more accuracy.
\end{abstract}

Keywords: Binary matrix, Geometric shape, Fire Bird V, Mat Lab, AVR studio.

\section{INTRODUCTION}

Robotics is the branch of technology that deals with the household maintenance (such as cleaning), waste water design, construction, operation, and application of robots, treatment and delivering goods and services. Some as well as computer systems for their control, sensory modern factory robots are "autonomous" within the strict feedback, and information processing. The design of a confines of their direct environment.[10] It may not be that given robotic system will often incorporate principles of every degree of freedom exists in their surrounding mechanical engineering, electronic engineering and environment, but the factory robot's workplace is computer science (particularly artificial intelligence). [7] challenging and can often contain chaotic, unpredicted The term 'robotics' was coined by Isaac Asimov in his variables. The exact orientation and position of the next science fiction short story called 'Liar'. Robot is an electro object of work and (in the more advanced factories) even mechanical machine which is guided by a electronic the type of object and the required task must be circuitry or computer program to perform various tasks. determined. This can vary unpredictably (at least from the [8] A robotic arm is a robotic manipulator, usually robot's point of view).One important area of robotics programmable, with functions similar to that of human research is to enable the robot to cope with its arm. [1]

Mobile Robotics: mobile robotics is an automatic machine that is cable of locomotion. Mobile robots have the capability to move around in their environment and are not fixed to one physical location. Mobile robots can be autonomous, which means they are cable of navigating an uncontrolled environment without the need for physical or mechanical guidance devices. [6]

Sensors: A sensor is an object whose purpose is to detect events is changes in its environment, and then provides corresponding output. A sensor is a type of transducer. Sensors may provide various types of output but typically use electrical or optical signals. For example, thermocouple generates a known voltage in response to its temperature. [9] Mercury in glass thermometer similarly converts measured temperature into expansion and contraction of a liquid which can read on calibrated glass tube. Sensors sensitivity indicates how much the sensors output changes when the input quantity being measured changes.

Autonomous robot is a robot that performs behaviors or tasks with a high degree of autonomy, which is particularly desirable in fields such as spaceflight, environment whether this be on land, underwater, in the air, underground, or in space.

A fully autonomous robot can

- Gain information about the environment.

- Work for an extended period without human intervention.

- Move either all or part of itself throughout its operating environment without human assistance.

- Avoid situations that are harmful to people, property, or itself unless those are part of its design specifications

- An autonomous robot may also learn or gain new knowledge like adjusting for new methods of accomplishing its tasks or adapting to changing surrounding

\section{FIRE BIRD V}

The Fire Bird V robot is the 5th in the Fire Bird series of robots. All the Fire Bird V series robots share the same main board and other accessories. Different family of microcontrollers can be added by simply changing top microcontroller adapter board. Fire Bird V supports ATMEGA2560 (AVR), P89V51RD2 (8051) and LPC2148 (ARM7) microcontroller adapter boards. This 
Vol. 5, Issue 12, December 2016

modularity in changing the microcontroller adapter boards Sensors:

makes Fire Bird V robots very versatile. We can also add Three white line sensors (extendable to 7)

your own custom designed microcontroller adapter Five Sharp GP2Y0A02YK IR range sensor (One in default board.[14]

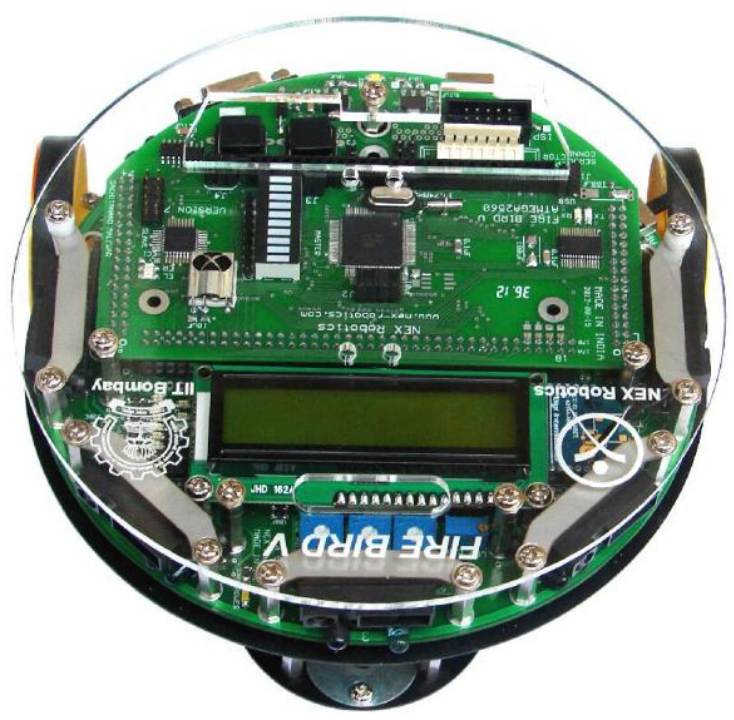

Fig 1: Fire Bird V ATMEGA2560 (AVR)

The figure1 shows a typical Fire Bird V robot, which is used in our project. Robot's electronics is static sensitive. When the battery of the robot drains out, then the buzzer starts beeping. Compatibility must be checked while interfacing any new hardware to the robot.

A typical Fire Bird V includes a number of components like motor, buzzer, sensors, battery, USB port, encoders, LCD etc. A block diagram showing the components of a typical fire bird $\mathrm{V}$ robot would help us understand about the robot in a better way.

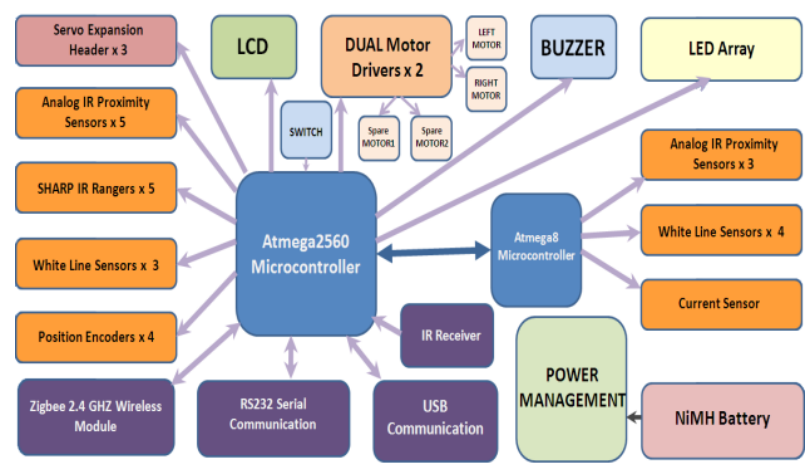

Fig 2: Fire Bird V ATMEGA2560 robot block diagram

The figure 2 shows the different components of the robot. The technical specification of Fire Bird V ATMEGA2560 is as given below.

\section{Microcontroller:}

Atmel ATMEGA2560 as Master microcontroller (AVR architecture based Microcontroller)

Atmel ATMEGA8 as Slave microcontroller (AVR architecture based Microcontroller) configuration)

Eight analog IR proximity sensors

Two position encoders (extendable to four)

Battery voltage sensing

Current Sensing (Optional)

Five Max Botix Ultrasonic Range Sensors (Optional)

\section{Indicators:}

2 x 16 Characters LCD

Buzzer and Indicator LEDs

Control:

Autonomous Control

PC as Master and Robot as Slave in wired or wireless mode

Communication:

USB Communication

Wired RS232 (serial) communication

Wireless ZigBee Communication (2.4GHZ) (if XBee wireless module is installed)

$\mathrm{Wi}-\mathrm{Fi}$ communication (if $\mathrm{Wi}-\mathrm{Fi}$ module is installed)

Bluetooth communication (if Bluetooth wireless module is installed)

Simplex infrared communication (From infrared remote to robot)

\section{Dimensions:}

Diameter: $16 \mathrm{~cm}$

Height: $8.5 \mathrm{~cm}$

Weight: $1100 \mathrm{gms}$

\section{Power:}

9.6V Nickel Metal Hydride (NiMH) battery pack and external Auxiliary power from battery Charger. On Board Battery monitoring and intelligent battery charger.

\section{Battery Life:}

2 Hours, while motors are operational at $75 \%$ of time

\section{Locomotion:}

Two DC geared motors in differential drive configuration and caster wheel at front as support

Top Speed: $24 \mathrm{~cm} /$ second

Wheel Diameter: $51 \mathrm{~mm}$

Position encoder: 30 pulses per revolution

Position encoder resolution: $5.44 \mathrm{~mm}$ [12]

Fire Bird V robot has 6 important modules:

1. Power management

2. Sensing

3. Actuation (locomotion)

4. Other peripherals

5. Communication

6. Intelligence (microcontroller)

\section{Sensors:}

Robot uses five sharp IR range sensors. These sensors consist of IR LED and linear CCD array [15]. It also uses Infrared proximity sensors which detect proximity of any obstacles in the short range. IR proximity sensors have about $10 \mathrm{~cm}$ sensing range. These sensors sense the presence of the obstacles in the blind spot region of the Sharp IR range sensors. Fire Bird V robot has 8 IR 
proximity sensors. When enabled 8 IR proximity sensors images which refers to this shades of gray is referred as combined together consumes about 51mA current. We can Grayscale [13][16]. The JPEG image, as shown in Figure save power by turning on theses sensors only when 4 is converted into Hex file. [2] Execution of the code in required. White line sensors are used for detecting white the MatLab generates the matrix as shown in the Figure 5. line on the ground surface. White lines are used to give The matrix is given as input to the AVR studio which will robot sense of localization. White line sensor consists of a intern execute the program and burn Hex file. The output highly directional photo transistor for line sensing and of Hex file is given to Firebird V. The movement of the bright red LED for the illumination. Fire Bird V robot can robot is based on the matrix [18]. In the matrix 1's is for be equipped with the 5 ultrasonic sensors from MaxBotix. the edges and 0's is for non-edges [5].Robot does the Each sensor can sense distance range from 6 inches to 254 movement from 1's to another 1's.

inches. Ultrasonic sensor transmits a narrow beam of ultrasonic pulse and measures time taken for echo of the beam. It gives output proportional to time taken for the ultrasonic beam to return echo from the obstacle.

\section{Buzzer:}

Robot has $3 \mathrm{KHz}$ piezo buzzer. It can be used for debugging purpose or as attention seeker for a particular event. The buzzer is connected to PC3 pin of the microcontroller. Also the same buzzer is used in battery monitoring circuit to alert the battery low indication.

\section{USB communication:}

Fire Bird V's main board has USB port socket. Microcontroller accesses USB port via main board socket. All its pins are connected to the microcontroller adapter board via main board's socket connector.

\section{PROPOSED METHOLOGY}

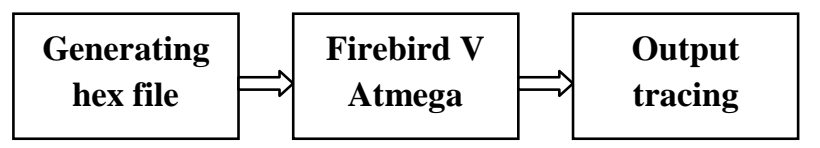

Fig.3 Block diagram of Drawing Robot

The figure 3 shows the block diagram Drawing Robot using Fire bird V.

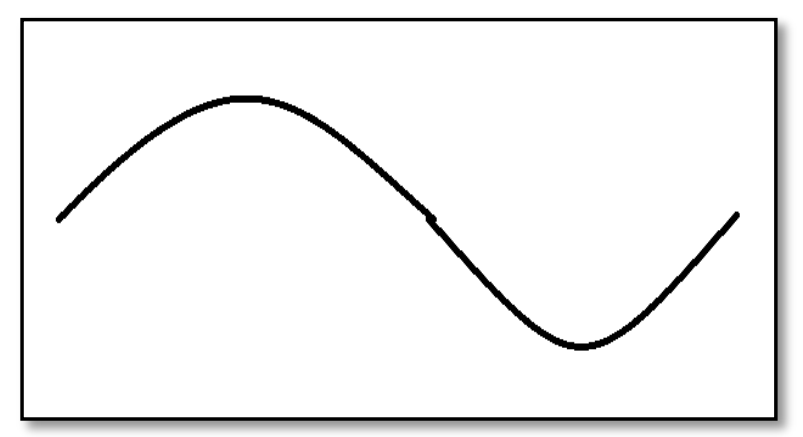

Fig.4 Input image

MatLab is a high level technical computing language and interactive environment for algorithm, development, data visualization and data analysis [3].

We are using image processing to process the input image. [17] Image processing is a processing of images using mathematical operations by using any form of signal processing for which input is the image, output may be image or the characteristics or parameters related to the image [4]. The input image is a Black-and-white photography actually consists of many shades of gray,
The movement of robot is based on some constraints, they are as follows:

a. nearest left 1

b. nearest top and bottom 1

c. nearest right[20]

Fire Bird V will help you get acquainted with the world of robotics and embedded systems. Firebird is an open source SQL relational database management (RDBMS) system that runs on Linux, Microsoft Windows and a variety of UNIX platforms. Firebird offers concurrency, high performance and powerful language supports for stored procedures and triggers. Ideal for doing research in the areas of robotics, embedded systems, artificial intelligence and sensor networks etc. Output of the image is drawn on a sheet of paper using pen or pencil [19]. It involves the selection of suitable motor for our application, deciding on the material to be used for the construction of the arm, i.e. the shaft material and deciding on the location where the motor has to be placed. A servomotor is a rotary actuator that allows for precise control of angular position, velocity and acceleration. It consists of a suitable motor coupled to a sensor for position feedback. It also requires a relatively sophisticated controller, often a dedicated module designed specifically for use with servomotors. To obtain high torque and precise speed servo motors are used.

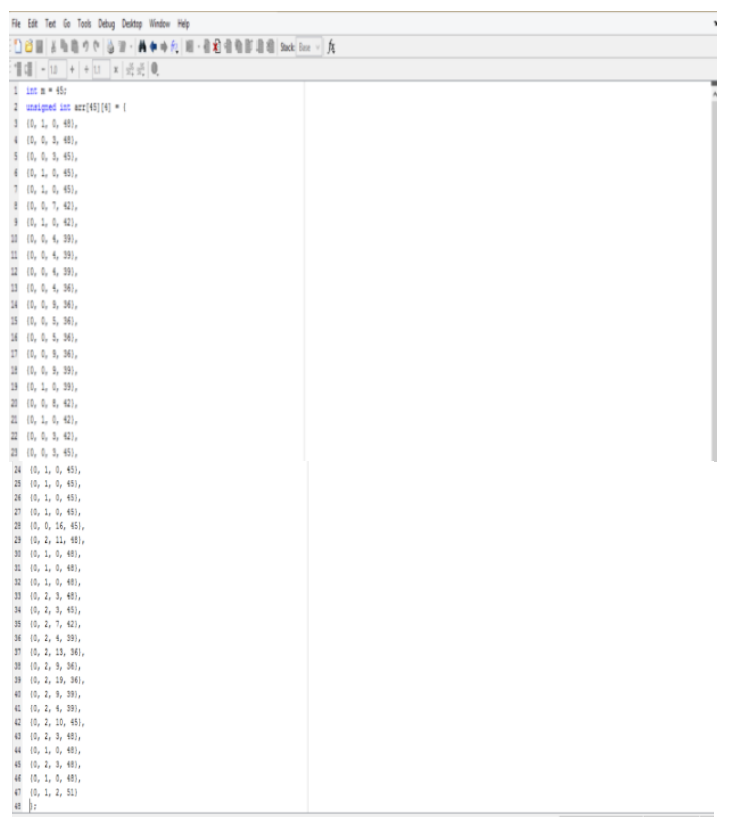

Fig.5 Generated hex values of the image 
Vol. 5, Issue 12, December 2016

\section{RESULTS AND DISCUSSIONS}

This project helps us to draw an image faster with more accuracy about $80 \%$. Robot has an ability to work at a constant speed without pausing for breaks. It is powered by high performance rechargeable battery.

The battery life is 2 Hours, while motors are operational at $75 \%$ of time. So it's difficult to work for longer duration. For better accuracy the algorithms must be more efficient.

\section{CONCLUSION}

Robo-Artist is an application which creates the drawing using Fire Bird V Robot through image processing. Drawing robot finds its application in many fields. It is a useful device in the field of graphics. Letters and images can be drawn by taking the inputs from the PC. Further, additional improvements can be done by incorporating the graphical user interface for making the arm more user could be controlled in remote place by the Web browser.

\section{REFRENCES}

[1]. Dr M Shivakumar, Stafford Michahail, Ankitha Tantry H, Bhawana C K, Kavana H, Kavya V Rao (Prof \& Head, Dept. Of TE, GSSIETW, Mysore, Asst. Prof Dept. of TE, GSSSIETW, Mysore, Students of TE, GSSSIETW, Mysore). "Robotic 2D Plotter" International Journal of Engineering and Innovative Technology (IJEIT) Volume 3, Issue 10, April 2014

[2]. Sowmya M et al | IJCSET (www.ijcset.net) | August 2016 | Vol 6, Issue 8, 299-301

[3]. Shutao Li et.al emphasizes in their entitled on "Multifocus Image fusion using artificial neural networks" published on June 2001.

[4]. Swathy Nair et.al discusses in their paper entitled "Pixel level image fusion using fuzzy let fusion algorithm", published on December 2013.

[5]. Michael Stokes et.al emphasizes in their book entitled "A Standard Default color Space for the Internet - sRGB", published on Dec 1897

[6]. J. Iijima, S. Asaka and S.Yuta, Yuta, "Searching Unknown Environment by a Mobile Robot Using Range Sensor-An Algorithm and Experiment-", Proc. IEEE/RSJ IROS 89, vol.1, pp.46-53, 1989.

[7]. R K Mittal, I J Nagrath, "Robotics and control", 2008, Tata Mcgraw Hill Education Private Limited.

[8]. MosheShoham: Textbook of Robotics: I Basic Concepts. Anchor Brendon Ltd.

[9]. Richard D. Klafter: Robotic Engineering. Prentice- Hall of India

[10]. Charles. A. Schuler, William L.McNamee: Industrial Electronics and Robotic. McGraw-Hill Book Company.

[11]. Schuler-McNamee: Industrial Electronics and Robotics. Tata McGraw-hill International Editions

[12]. Dr.Bindu A Thomas, Stafford Michahial, Shreeraksha P, Vijayashree B, Suresh M, "Industry based automatic Robotic arm", in IJEIT, Vol 2, 2013.

[13]. Stephen Johnson describes in the paper entitled "Stephen Johnson on Digital Photography", published on 2006.

[14]. ERTS lab. CSE, IIT Bombay www.it.iitb.ac.in/ erts

[15]. S K Saha, "Introduction to robotics", 2008, Tata McGraw Hill Education Private Limited

[16]. "A Humanoid Robot Drawing Human Portraits"Sylvain Calinon, Julien Epiney and Aude Billard Ecole Polytechnique F'ed'erale de Lausanne (EPFL), CH-1015 Lausanne, Switzerland \{sylvain.calinon, julien.epiney, aude.billard\} @epfl.ch Proceedings of 2005 5th IEEE-RAS International Conference on Humanoid Robots.

[17]. Charles Poynton discusses in the paper entitled "Image Acquisition”, published on 1998.
[18]. Swathy Nair et.al discusses in their paper entitled "Pixel level imagefusion using fuzzy let fusion algorithm", published on December 2013.

[19]. Anish.T describes in the book entitled on "A survey on multifocus image fusion methods", published on October 2012.

[20]. Muthukrishnan R, M.Radha(2011). Edge detection techniques for image segmentation, International Journal of Computer Science and Information Technology (IJCSIT) Vol.3, No.6, pp.250-254. 